\title{
Frequency-dependent specific heat from thermal effusion in spherical geometry
}

Jakobsen, Bo; Olsen, Niels Boye; Christensen, Tage Emil

Published in:

Physical Review E (Statistical, Nonlinear, and Soft Matter Physics)

DOI:

10.1103/PhysRevE.81.061505

Publication date:

2010

Document Version

Publisher's PDF, also known as Version of record

Citation for published version (APA):

Jakobsen, B., Olsen, N. B., \& Christensen, T. E. (2010). Frequency-dependent specific heat from thermal effusion in spherical geometry. Physical Review E (Statistical, Nonlinear, and Soft Matter Physics), 81(6), 061505. https://doi.org/10.1103/PhysRevE.81.061505

\section{General rights}

Copyright and moral rights for the publications made accessible in the public portal are retained by the authors and/or other copyright owners and it is a condition of accessing publications that users recognise and abide by the legal requirements associated with these rights.

- Users may download and print one copy of any publication from the public portal for the purpose of private study or research.

- You may not further distribute the material or use it for any profit-making activity or commercial gain.

- You may freely distribute the URL identifying the publication in the public portal.

\section{Take down policy}

If you believe that this document breaches copyright please contact rucforsk@kb.dk providing details, and we will remove access to the work immediately and investigate your claim. 


\title{
Frequency-dependent specific heat from thermal effusion in spherical geometry
}

\author{
Bo Jakobsen, ${ }^{*}$ Niels Boye Olsen, and Tage Christensen ${ }^{\dagger}$ \\ DNRF Centre "Glass and Time," IMFUFA, Department of Sciences, Roskilde University, Postbox 260, DK-4000 Roskilde, Denmark
}

(Received 23 April 2010; published 25 June 2010)

\begin{abstract}
We present a method of measuring the frequency-dependent specific heat at the glass transition applied to 5-polyphenyl-4-ether. The method employs thermal waves effusing radially out from the surface of a spherical thermistor that acts as both a heat generator and a thermometer. It is a merit of the method compared to planar effusion methods that the influence of the mechanical boundary conditions is analytically known. This implies that it is the longitudinal rather than the isobaric specific heat that is measured. As another merit the thermal conductivity and specific heat can be found independently. The method has highest sensitivity at a frequency where the thermal diffusion length is comparable to the radius of the heat generator. This limits in practice the frequency range to 2-3 decades. An account of the $3 \omega$ technique used including higher-order terms in the temperature dependence of the thermistor and in the power generated is furthermore given.
\end{abstract}

DOI: 10.1103/PhysRevE.81.061505

PACS number(s): 64.70.P-

\section{INTRODUCTION}

A hallmark of the glass transition of supercooled liquids is the time dependence of physical properties-also called relaxation. This time dependence is typically seen on the time scale of the so-called Maxwell relaxation time $\tau_{M}$. It is defined as

$$
\tau_{M}=\frac{\eta_{0}}{G_{\infty}},
$$

where $\eta_{0}$ is the low-frequency limiting (dc) shear viscosity and $G_{\infty}$ is the high-frequency limiting elastic shear modulus. The relaxation is often most easily studied in the frequency domain. At high frequencies the liquid shows solidlike elastic behavior whereas at low frequencies it shows liquidlike viscous behavior. In general the viscoelastic stress response to oscillatoric shear strain is described by the complex dynamic shear modulus $G$ [1]. The structural relaxation is also observed in the specific heat. This has long ago been studied in the time domain as enthalpy relaxation [2], but in 1985 frequency domain studies or ac calorimetry $[3,4]$ was introduced in the research field of supercooled liquids. Common to these methods is that they excite thermal oscillations in the sample at a cyclic frequency $\omega$. For a heat diffusivity $D$, this frequency is associated with a characteristic heat diffusion length,

$$
l_{D}=\sqrt{\frac{D}{i \omega}},
$$

the magnitude of which gives the range of the excited thermal waves. When comparing $\left|l_{D}\right|$ to a given sample size $L$, one can classify ac-calorimetry experiments as to whether the sample is thermally thin $\left(L \ll\left|l_{D}\right|\right)$ or thermally thick $(L$ $\left.\gg\left|l_{D}\right|\right)$. A typical value of $D$ is $0.1 \mathrm{~mm}^{2} / \mathrm{s}$ giving $\left|l_{D}\right|$ $\approx 0.1 \mathrm{~mm}$ for a frequency of $\nu=\omega /(2 \pi)=1 \mathrm{~Hz}$. This means that thermally thin methods are restricted to rather low fre-

\footnotetext{
*boj@ruc.dk

†tec@ruc.dk
}

quencies $[4,5]$ or very thin samples [5,6]. In these methods the temperature field is homogeneous throughout the sample and the frequency-dependent specific heat is derived directly. The ac technique allows for easy corrections of the influence of heat leaks in a nonadiabatic configuration. ac calorimetry on thermally thick samples on the other hand has the advantage of covering a large frequency span $[3,7]$. In this case the temperature field is inhomogeneous and what is really measured is the effusivity $e=\sqrt{\lambda c}$, from which the specific heat is derived. The effusivity expresses the ability of the liquid to take up heat from parts of its surface and transport it away (thermal effusion). This ability is dependent on two constitutive properties: the heat conductivity $\lambda$ and the specific heat (per volume) $c$. The specific heat can only be found to within a proportionality constant in effusion experiments from an (infinite) plane. However, taking boundary effects into account for finite-size plane heaters $[8,9]$ one may in principle find $\lambda$ and $c$ independently. The intermediate situation where the heat diffusion length is comparable to the sample thickness has been exploited in the so-called twochannel ac calorimeter $[10,11]$. This fine method aims directly at an independent determination of $\lambda$ and $c$.

The specific heat entering the effusivity is widely taken as the isobaric specific heat $c_{p}$. However, recently it has been shown [12-14] that in planar and spherical geometries it is rather the longitudinal specific heat $c_{l}$ that enters. When $M_{S}$ and $M_{T}$ are the adiabatic and isothermal longitudinal moduli, respectively, $c_{l}$ is related to the isochoric specific heat $c_{V}$ by

$$
c_{l}=\frac{M_{S}}{M_{T}} c_{v} .
$$

In contrast, when $K_{S}$ and $K_{T}$ are the adiabatic and isothermal bulk moduli, respectively, $c_{p}$ is given by

$$
c_{p}=\frac{K_{S}}{K_{T}} c_{v} .
$$

Now $M_{S}=K_{S}+4 / 3 G$ and $M_{T}=K_{T}+4 / 3 G$. This means that $c_{l}$ will differ from $c_{p}$ in the relaxation regime where the shear modulus $G$ dynamically is different from zero, and $K_{s}$ and $K_{T}$ differ significantly. 
In this work we study the frequency-dependent specific heat from thermal effusion in a spherical geometry. Specificheat measurements in a spherical geometry have earlier been conducted in the thermal thin limit [5], but in this paper we explore the thermal thick limit.

The justification is threefold. (1) We want to realize an experimental geometry in which the thermomechanical influence on the thermal effusion can be analytically calculated [14]. (2) The spherical container of the liquid is a piezoelectric spherical shell by which it is possible to measure the adiabatic bulk modulus [15] on the very same sample under identical conditions. (3) The heat conductivity and specific heat can be found independently, as the introduction of a finite length scale allows for separation of the two. We also in this paper give an account of the $3 \omega$-detection technique of our variant.

\section{LINEAR-RESPONSE THEORY APPLIED TO THERMAL EXPERIMENTS}

The experimental method used in this study is an effusion method. In such methods a harmonic varying heat current $\operatorname{Re}\left(P e^{i \omega t}\right)$, with angular frequency $\omega$ and complex amplitude $P$, is produced at a surface in contact with the liquid; this heat then "effuses" into the liquid. At the surface a corresponding harmonic temperature oscillation $\operatorname{Re}\left(\delta T e^{i \omega t}\right)$, with complex amplitude $\delta T$, is created. The current and temperature can be thought of as stimuli and response (but which is which can be interchanged at will), and the two are proportional if the amplitudes $|P|$ and $|\delta T|$ are small enough. Hence, it is convenient to introduce the complex frequencydependent thermal impedance of the liquid,

$$
Z_{\text {liq }}=\frac{\delta T}{P} \text {. }
$$

This impedance depends on the properties of the liquid.

The terminology thermal impedance derives naturally from the close analogy between the flow of thermal heat and the flow of electricity [16]. Temperature corresponds to electric potential and heat current to electrical current. The analogy also applies to the heat conductivity which corresponds to the specific electrical conductivity and to the specific heat which corresponds to electrical capacity.

As an example the thermal impedance of a thermally thin sample is given as

$$
Z_{\text {liq, thin }}=\frac{1}{i \omega c V},
$$

where $V$ is the volume of the sample, again an equation completely equivalent to the electrical correspondence between capacitance and impedance. In the classical case of a planar heater in a thermal thick situation the thermal impedance is found to be [3]

$$
Z_{\text {liq,planar }}=\frac{1}{A \sqrt{i \omega c \lambda}},
$$

where $A$ is the plate area. The lateral dimension $W$ has to be large $\left[W \gg\left|l_{D}(\omega)\right|\right]$ for this formula to be exact and unfortu- nately correction terms that take boundary effects into account decay only slowly as $\omega^{-1 / 2}$ [9].

\section{THERMAL EFFUSION IN SPHERICAL GEOMETRY}

Thermal effusion is possible in a spherical geometry as well as in a planar geometry. Imagine a liquid confined between two radii $r_{1}$ and $r_{2}$. The imposed oscillating outward heat current $\operatorname{Re}\left[P\left(r_{1}\right) e^{i \omega t}\right]$ at radius $r_{1}$ results in a temperature oscillation $\operatorname{Re}\left[\delta T\left(r_{1}\right) e^{i \omega t}\right]$ at the same radius. The heat wave propagating out into the liquid gives rise to a coupled strain wave due to thermal expansion. The heat diffusion is thus dependent on the mechanical boundary conditions and mechanical properties of the liquid. It is widely assumed that the transport of heat in a continuum is described by the heat diffusion equation

$$
i \omega \delta T=D \nabla^{2} \delta T .
$$

By the same token one might take the heat diffusion constant as $D=\lambda / c_{v}$ if the surfaces at $r_{1}$ and $r_{2}$ are clamped and $D$ $=\lambda / c_{p}$ if one of the surfaces is free to move. This common belief is not entirely correct. If shear modulus is nonvanishing compared to bulk modulus the description of heat diffusion cannot be decoupled to a bare heat diffusion equation [12-14,17]. Such a situation arises dynamically at frequencies where $\omega \approx 1 / \tau_{M}$. The fully coupled thermomechanical equations have been discussed in detail, by some of us, in planar geometry [12] and in spherical geometry [14]. In general the solutions are complicated but in the case of a spherical geometry where the outer radius $r_{2}$ is much larger than the thermal diffusion length, $\left|l_{D}(\omega)\right| \ll r_{2}$, the solution becomes simple. In terms of the liquid thermal impedance $Z_{\text {liq }}$, at the inner radius $r_{1}$, one has

$$
Z_{\text {liq }}=\frac{\delta T\left(r_{1}\right)}{P\left(r_{1}\right)}=\frac{1}{4 \pi \lambda r_{1}\left(1+\sqrt{i \omega r_{1}^{2} c_{l} / \lambda}\right)} .
$$

It is very interesting that in this thermally thick limit, the thermal impedance is independent of the mechanical boundary conditions $[13,14]$. That is, in the two cases of clamped or free boundary conditions mentioned before it is neither the isochoric nor the isobaric specific heat that enters the diffusion constant and the thermal impedance but the longitudinal specific heat. Since a closed spherical surface has no boundary there are no correction terms like the ones to Eq. (7) discussed above for planar plate effusion. Notice from Eq. (9) that associated with a given radius $r_{1}$ is a characteristic heat diffusion time for the liquid,

$$
\tau_{l}=r_{1}^{2} c_{l} / \lambda .
$$

$c_{l}$ and hence $\tau_{l}$ may in general be complex, but when $c_{l}$ is real, the imaginary part of $Z_{\text {liq }}$ peaks at $\omega=1 / \tau_{l}$.

\section{IV. $3 \omega_{\mathrm{e}}$-DETECTION TECHNIQUE}

The $3 \omega_{\mathrm{e}}$-detection technique we use (where the subscript "e" indicates that it is the electrical angular frequency not the thermal one) is different from other $3 \omega_{\mathrm{e}}$ techniques (e.g., Ref. [18]) in two ways. First, we include higher-order terms 
in the temperature dependence of the thermistor used and in the power oscillations produced. Second, we use a direct measurement of the time-dependent voltage over a voltage divider instead of a lock-in amplifier.

\section{A. Principle}

The $3 \omega_{\mathrm{e}}$-detection technique makes it possible to measure the temperature with the same electrical resistor which generates the heat and thereby to know the thermal current and the temperature at the same surface. The principle is in short the following: applying an electrical current at cyclic frequency $\omega_{\mathrm{e}}$ through a resistor a combined constant (zerofrequency) and $2 \omega_{\mathrm{e}}$ component Joule heating is produced. This gives rise to a combined temperature dc offset and temperature oscillation at $2 \omega_{\mathrm{e}}$, the size of which depends on the thermal environment (the thermal impedance). The resistor is chosen to be temperature dependent; it is a so-called thermistor. The temperature oscillation therefore creates a perturbation of the resistance at frequency $2 \omega_{\mathrm{e}}$. The voltage across the thermistor which by Ohm's law is the product of current and resistance thus contains a $3 \omega_{\mathrm{e}}$ (and an additional $\omega_{\mathrm{e}}$ ) component proportional to the temperature oscillation at $2 \omega_{\mathrm{e}}$. In practice one rather has a voltage source than a current source. In order to get a $3 \omega_{\mathrm{e}}$ signal the thermistor is therefore placed in series with a temperature-independent preresistor as detailed in the following.

The result of the analysis is the frequency-dependent thermal impedance at a frequency of $2 \omega_{\mathrm{e}}$. It is convenient to express this thermal impedance as function of the frequency of the thermal signal which we designate $\omega$ and notice that $\omega=2 \omega_{\mathrm{e}}$.

We first outline the simplest theory in order to elucidate the technique most clearly. However, to increase the signalto-noise ratio it is expedient to choose a thermistor with a large temperature dependence and to choose temperature amplitudes as high as possible although within the linear regime of the liquid thermal response. This necessitates higher harmonics be taken into account. This detailed analysis is deferred to Appendix A.

\section{B. Detour on the complex notation}

Complex numbers are known to be of great use in harmonic analysis of linear systems. Since the heat current is quadratic in the imposed electrical current and the whole idea of the $3 \omega_{\mathrm{e}}$-detection technique is to exploit the inherent nonlinearity introduced by the temperature dependence of the thermistor, the formalism becomes a little more troubled than in pure linear applications. Thus, we have to drag around the complex conjugate terms in order to get product terms right.

Generally a sum of harmonic terms,

$$
A=A_{0}+\left|A_{1}\right| \cos \left(\omega_{\mathrm{e}} t+\phi_{1}\right)+\cdots+\left|A_{n}\right| \cos \left(n \omega_{\mathrm{e}} t+\phi_{n}\right),
$$

with real amplitudes $A_{0},\left|A_{k}\right|$ and phases $\phi_{k}$, can be written as

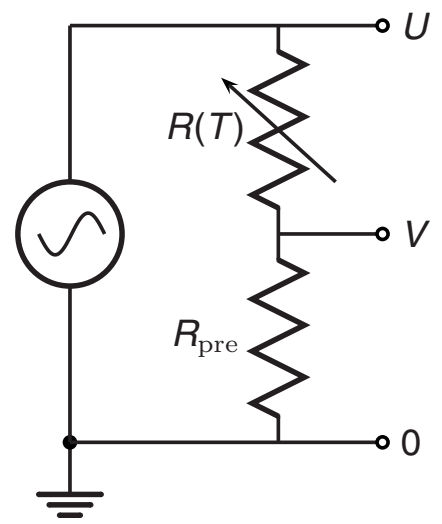

FIG. 1. Voltage divider for the $3 \omega_{\mathrm{e}}$-detection technique. The temperature-dependent resistor (the thermistor) $R(T)$ is in thermal contact with the liquid probing its thermal impedance at $2 \omega_{\mathrm{e}}$. The preresistor $R_{\text {pre }}$ is temperature independent.

$$
\begin{aligned}
A= & \frac{1}{2}\left(A_{0}+A_{0}+\left|A_{1}\right| e^{i\left(\omega_{\mathrm{e}} t+\phi_{1}\right)}+\left|A_{1}\right| e^{-i\left(\omega_{\mathrm{e}} t+\phi_{1}\right)}+\cdots\right. \\
& \left.+\left|A_{n}\right| e^{i\left(n \omega_{\mathrm{e}} t+\phi_{n}\right)}+\left|A_{n}\right| e^{-i\left(n \omega_{\mathrm{e}} t+\phi_{n}\right)}\right) .
\end{aligned}
$$

By introducing the complex amplitudes $A_{k}=\left|A_{k}\right| e^{i \phi_{k}}$ and the shorthand notation $E_{k}=e^{i k \omega_{\mathrm{e}} t}$, the sum becomes

$$
A=\frac{1}{2}\left(A_{0}+A_{1} E_{1}+\cdots+A_{n} E_{n}+\text { c.c. }\right),
$$

where +c.c. means that the complex conjugated of all the terms within the parenthesis should be added, including the real constant term. Note that $E_{0}=1, E_{k} E_{l}=E_{k+l}$, and $E_{k} E_{l}^{*}$ $=E_{k-l}$.

Notice that any product of $n$th and $m$ th harmonics will produce both $(n+m)$ th and $(n-m)$ th harmonic terms. Furthermore, the dc components get doubled because there is no difference between $E_{0}$ and $E_{0}^{*}$.

\section{Fundamental equations for the voltage divider}

Consider the diagram of the electrical setup seen in Fig. 1. $U(t)$ is the voltage of the source and $V(t)$ is the voltage across the temperature-independent preresistor $R_{\text {pre }} . U(t)$ and $V(t)$ can be measured directly. $R(T(t))$ is the temperaturedependent resistance of the heater/sensor [we will explicitly state the temperature (time) dependencies when appropriate; otherwise, it is implicitly assumed].

The current $I(t)$ through $R(T(t))$ and $R_{\text {pre }}$ is given as

$$
I(t)=\frac{U(t)}{R_{\text {pre }}+R(T(t))}=\frac{V(t)}{R_{\text {pre }}},
$$

and the power produced in the heater is

$$
P(t)=I(t)^{2} R(T(t))=\frac{R(T(t))}{\left[R_{\mathrm{pre}}+R(T(t))\right]^{2}} U(t)^{2} .
$$

Equation (14) gives the voltage divider equation,

$$
V(t)=\frac{R_{\mathrm{pre}}}{R_{\mathrm{pre}}+R(T(t))} U(t),
$$

which in principle allows for determination of $T(t)$ from the measured voltages. 
TABLE I. Experimental parameters. Top: parameters characterizing the resistors in the setup; $R_{\text {pre }}$ is the resistance of the preresistor, and $R_{\infty}$ and $T_{a}$ are the characteristics of the thermistor according to Eq. (19). Middle: absolute size of the thermistor bead. Measured values indicate the eccentricity of the bead. The optimized value is the one use in the final treatment of the data and is optimized to give best correspondence between the room-temperature dc specific heat and the literature value of this. Bottom: applied amplitudes expressed as mean applied voltage $\left(\left\langle\left|U_{1}\right|\right\rangle_{\nu}\right)$ and maximum temperature amplitude $\left[\max \left(\left|T_{2}\right|\right)_{\nu, T}\right]$.

\begin{tabular}{lc}
\hline \hline$R_{\text {pre }}$ & $3884.3 \Omega$ \\
$R_{\infty}$ & $0.1251 \Omega$ \\
$T_{a}$ & $2789.5 \mathrm{~K}$ \\
& \\
$r_{1, \text { Measured, long edge }}$ & $0.205 \mathrm{~mm}$ \\
$r_{1, \text { Measured, short edge }}$ & $0.185 \mathrm{~mm}$ \\
$r_{1, \text { Optimized }}$ & $0.2073 \mathrm{~mm}$ \\
$\left\langle\left|U_{1}\right|\right\rangle_{\nu}$ & \\
$\max \left(\left|T_{2}\right|\right)_{\nu, T}$ & $4.909 \mathrm{~V}$ \\
\hline \hline
\end{tabular}

\section{D. ac method to lowest order}

The thermistor and the surrounding sample are placed in a cryostat that defines an overall reference temperature $T_{\text {cryo }}$. The power produces a (real) temperature change $\Delta T=T$ $-T_{\text {cryo }}$ at the heater/sensor depending on the thermal impedance $Z$ (as defined in Sec. II) the heater/sensor looks into. The temperature rise has a real dc component $T_{0}$ and complex ac components. It can in general be written as

$$
\Delta T=\frac{1}{2}\left(T_{0}+T_{1} E_{1}+T_{2} E_{2}+T_{3} E_{3}+T_{4} E_{4}+\cdots+\text { c.c. }\right) .
$$

The dominant terms will be the dc component $T_{0}$ and the second harmonic term $T_{2}$ (see the next section).

To first order in the temperature variations the thermistor has a resistance of

$$
R=R_{0}\left(1+\alpha_{1} \Delta T\right) \text {. }
$$

Here, $R_{0}$ and $\alpha_{1}$ are considered constant at a given cryostat temperature but they vary with $T_{\text {cryo }}$. In fact the thermistor, which is based on a semiconducting material, has a resistance that over a larger temperature range is very well described by

$$
R=R_{\infty} e^{T_{a} / T},
$$

with $T_{a}$ as the activation temperature and $R_{\infty}$ as the infinitetemperature limiting resistance. $\alpha_{1}$ is related to $T_{a}$ by

$$
\alpha_{1}=\left.\frac{1}{R\left(T_{\text {cryo }}\right)} \frac{d R}{d T}\right|_{T_{\text {cryo }}}=-\frac{T_{a}}{T_{\text {cryo }}^{2}} .
$$

Characteristic values of $R_{\infty}$ and $T_{a}$ are given in Table I. Substituting Eq. (18) into Eq. (16) we obtain

$$
V=\frac{1}{1+A\left(1+\alpha_{1} \Delta T\right)} U \approx \frac{1}{A+1}(1-a \Delta T) U,
$$

where $A=R_{0} / R_{\text {pre }}, a=A \alpha_{1} /(1+A)$, and the second expression is to first order in $\Delta T$ like in Eq. (18). In the simple case, which we discuss first, we assume that the function generator can deliver a pure single harmonic voltage,

$$
U(t)=\frac{1}{2}\left(U_{1} E_{1}+\text { c.c. }\right) \text {. }
$$

Equations (17) and (22) can now be substituted into Eq. (21),

$$
\begin{aligned}
V= & \frac{1}{A+1}\left[1-a\left(\frac { 1 } { 2 } \left(T_{0}+T_{1} E_{1}+T_{2} E_{2}+T_{3} E_{3}+T_{4} E_{4}+\cdots\right.\right.\right. \\
& + \text { c.c. }))] \frac{1}{2}\left(U_{1} E_{1}+U_{1}^{*} E_{1}^{*}\right) .
\end{aligned}
$$

In principle the result contains all harmonics, but it turns out that the interesting ones are the first and third harmonics. The complex amplitudes are by inspection seen to be

$$
\begin{gathered}
V_{1}=\frac{1}{A+1}\left(U_{1}-a T_{0} U_{1}-\frac{1}{2} a T_{2} U_{1}^{*}\right), \\
V_{3}=\frac{1}{A+1}\left(-\frac{1}{2} a T_{2} U_{1}-\frac{1}{2} a T_{4} U_{1}^{*}\right) .
\end{gathered}
$$

Notice that even if $\Delta T$ is written as an infinite sum [as in Eq. (17)], $V_{1}$ and $V_{3}$ have only the included terms. This is a consequence of assuming a perfect voltage source.

The $T_{4}$ term can be neglected since it is proportional to a higher-order power term $P_{4}$, which is small. This approximation leads to a useful relationship between the $3 \omega_{\mathrm{e}}$ term and the temperature amplitude at $2 \omega_{e}, T_{2}$,

$$
V_{3}=-\frac{1}{2} \frac{a}{(A+1)} T_{2} U_{1} \text {. }
$$

Equations (24) and (26) can be solved for $T_{0}$ and $T_{2}$ if prior knowledge on the temperature dependence of the resistance of the thermistor exists, that is, if $A$ and $a$ are known at the relevant temperatures. The $T_{2}$ and $T_{0}$ component terms are related to the power through the complex thermal acimpedance $Z_{2}$ (the impedance at $2 \omega_{\mathrm{e}}$ ) and thermal dcimpedance $Z_{0}$ as

$$
T_{0}=Z_{0} P_{0}, \quad T_{2}=Z_{2} P_{2} .
$$

The temperature dependence of the thermistor resistance can of course be found from calibration measurements. We have however found it to be more efficient to use an iterative solution technique eliminating the need for prior calibration. Equations (24) and (26) are rewritten in the following form:

$$
\begin{gathered}
A=\frac{\left(U_{1}-a T_{0} U_{1}-\frac{1}{2} a T_{2} U_{1}^{*}\right)}{V_{1}}-1, \\
T_{2}=-2 \frac{V_{3}(A+1)}{a U_{1}} .
\end{gathered}
$$

It can be seen that the equation for $A$ consists of the zerothorder expression $A \approx U_{1} / V_{1}-1$ which would be valid if the 
temperature of the thermistor bead was the same as the cryostat temperature. This is not the case as the thermistor generates self-heat, which is what the two additional terms correct for.

$A$ and $T_{2}$ can be found by iteration in the following way. A first approximation of $A$ is found by setting $T_{0}=0$ and $T_{2}$ $=0$ in Eq. (28a). Based on this an initial estimate of $\alpha_{1}$ is found from the temperature dependence of $A$. An estimate on $T_{2}$ (and hence $Z_{2}$ ) is afterward found from Eq. (28b). By extrapolating $Z_{2}$ to zero frequency an estimate of $T_{0}$ is found [19] (utilizing $T_{0}=Z_{0} P_{0}$ ). The estimated values for $T_{0}, T_{2}$ and $a$ can then be inserted into Eq. (28a) giving a better estimate for $A$. This iteration procedure can be repeated a number of times and converges rapidly to a fixed point for $A$ and $T_{2}$.

\section{E. Thermal-power terms}

If the first-order approximation of the resistance of the thermistor [Eq. (18)] is substituted into the power equation [Eq. (15)], the following expression is obtained to first order in $\alpha_{1} \Delta T$ :

$$
\begin{aligned}
P & =\frac{1}{R_{\text {pre }}} \frac{A\left(1+\alpha_{1} \Delta T\right)}{\left[1+A\left(1+\alpha_{1} \Delta T\right)\right]^{2}} U^{2} \\
& \approx \frac{1}{R_{\text {pre }}} \frac{A}{(1+A)^{2}}\left(1+\frac{1-A}{1+A} \alpha_{1} \Delta T\right) U^{2}
\end{aligned}
$$

This shows that an input voltage which is a purely single harmonic leads to dominant zero and double harmonics in the power and temperature. Notice that if the thermistor resistance were temperature independent, that is, $\alpha_{1}=0$, then $P_{0}$ equals $\left|P_{2}\right|$ exactly but in general they are almost identical, $P_{0} \approx\left|P_{2}\right|$.

It can further be seen that even if the voltage generator is a purely single harmonic, the power can have a $4 \omega_{\mathrm{e}}$ component from the interplay between the $2 \omega_{\mathrm{e}}$ temperature variation and the $2 \omega_{\mathrm{e}}$ variation of the squared voltage. In principle further higher harmonics are generated this way. It can however be observed that if $\alpha_{1} \Delta T$ is small, or if $A \approx 1$ which can be chosen in the setup, the higher harmonics will be small (and decreasing with order). Equation (29) is not very practical for calculating the thermal-power terms needed for evaluating the thermal impedance from the temperature amplitudes obtained from Eqs. (28) since $\Delta T$ is needed.

Alternatively the thermal power can be written as

$$
P=I(U-V)=\frac{(U-V) V}{R_{\mathrm{pre}}}
$$

using Eq. (14). From this equation the power can be directly determined from measurable quantities.

If only the $\omega_{\mathrm{e}}$ component of the voltage is taken into account the following approximations are obtained:

$$
\begin{aligned}
P & \approx \frac{1}{4 R_{\mathrm{pre}}}\left[\left(U_{1}-V_{1}\right) E_{1}+\text { c.c. }\right]\left(V_{1} E_{1}+\text { c.c. }\right) \\
& =\frac{1}{4 R_{\mathrm{pre}}}\left[\left(U_{1}-V_{1}\right) V_{1}^{*}+\left(U_{1}-V_{1}\right) V_{1} E_{2}+\text { c.c. }\right],
\end{aligned}
$$

leading to power coefficients given as

$$
\begin{gathered}
P_{0}=\frac{1}{4 R_{\text {pre }}}\left[\left(U_{1}-V_{1}\right) V_{1}^{*}+\left(U_{1}^{*}-V_{1}^{*}\right) V_{1}\right], \\
P_{2}=\frac{1}{2 R_{\text {pre }}}\left(U_{1}-V_{1}\right) V_{1} .
\end{gathered}
$$

A more general expansion including higher harmonics is given in Appendix B.

\section{F. ac method refined}

As mentioned earlier we have to do a refined analysis of the detected harmonics of the voltages. These extra terms stem from different sources: (1) The voltage source itself has higher harmonics and a dc offset. (2) A second-order term in the temperature dependence of the thermistor resistance. (3) The $4 \omega_{\mathrm{e}}$ Joule-power component produced by the $2 \omega_{\mathrm{e}}$ variation of the thermistor (as discussed in Sec. IV E). These effects are taken into account, as described below.

Regarding point (1) we write the source voltage $U(t)$ more generally as

$$
U(t)=\frac{1}{2}\left(U_{0}+U_{1} E_{1}+U_{2} E_{2}+U_{3} E_{3}+U_{4} E_{4}+\text { c.c. }\right),
$$

including up to the fourth-order harmonics. A $U_{3}$ component from the source is to be expected since the source itself has an inner resistance. $U_{0}$ is a dc offset that was present in this experiment but in principle should be eliminated.

Regarding point (2) we expand the temperature dependence of the thermistor to second order as

$$
R=R_{0}\left[1+\alpha_{1} \Delta T(t)+\alpha_{2} \Delta T(t)^{2}\right] .
$$

Because $R(T)$ follows Eq. (19) over an extended temperature range, $\alpha_{2}$ and $\alpha_{1}$ are connected as

$$
\alpha_{2}=\frac{\alpha_{1}^{2}}{2}-\frac{\alpha_{1}}{T_{\text {cryo }}} .
$$

Point (3) is taken into account by expanding $V(t), P(t)$, and $T(t)$ like $U(t)$ in Eq. (33) up to the fourth harmonic components also.

The full analysis can be found in Appendix A, including terms larger than $5 \times 10^{-7} U_{1}$. Fortunately it leads to rather simple extensions of Eqs. (28),

$$
\begin{gathered}
A=\frac{\left[U_{1}-a T_{0} U_{1}-\frac{1}{2} a T_{2} U_{1}^{*}+X_{1}\right]}{V_{1}}-1, \\
T_{2}=-2 \frac{V_{3}(A+1)-\left(U_{3}+X_{3}\right)}{a U_{1}} .
\end{gathered}
$$

$X_{1}$ and $X_{2}$ depend on $T_{0}, T_{1}, T_{2}, T_{4}$, and $b=\left[A \alpha_{1} /(1+A)\right]^{2}$ $-A \alpha_{2} /(1+A)$, and the expressions are given in Eqs. (A3). 
The coupled equations (36) can be solved by iteration, using a procedure equivalent to the one for the first-order solution. We start by estimating $A(T)$ from Eq. (36a), putting $X_{1}=T_{0}=T_{2}=0$, and from this estimate $\alpha_{1}$ and $\alpha_{2}$.

A provisional thermal impedance $Z_{2}$ is then found from Eq. (36b). $T_{1}=Z_{1} P_{1}$ and $T_{4}=Z_{4} P_{4}$ can then be estimated since $Z_{1}(\omega)=Z_{2}(\omega / 2)$ and $Z_{4}(\omega)=Z_{2}(2 \omega)$, and $T_{0}$ is found from the limit of $Z_{0}$ as described earlier. The perturbative terms $X_{1}, X_{3}$ can then be found and with these values in Eqs. (36a) and (36b) a better estimation of $A$ (and hence $\alpha_{1}$ and $\alpha_{2}$ ) and $T_{2}$ is found. This process is iterated until convergence.

The two important power components are calculated from $V_{i}$ 's and $U_{i}$ 's as in Eqs. (32) by

$$
P_{0}=\frac{1}{R_{\text {pre }}}\left(W_{0} V_{0}+\frac{1}{2} \operatorname{Re}\left(W_{1} V_{1}^{*}+W_{2} V_{2}^{*}+W_{3} V_{3}^{*}+W_{4} V_{4}^{*}\right)\right),
$$

$$
\begin{aligned}
P_{2}= & \frac{1}{R_{\mathrm{pre}}}\left(W_{0} V_{2}+W_{2} V_{0}+\frac{1}{2}\left(W_{1} V_{1}+W_{1}^{*} V_{3}+W_{2}^{*} V_{4}+W_{3} V_{1}^{*}\right.\right. \\
& \left.\left.+W_{4} V_{2}^{*}\right)\right)
\end{aligned}
$$

where $W=U-V$. The full set of power components is given in Eqs. (B1).

\section{EXPERIMENT}

The measurements were performed using a custom-built setup $[20,21]$. The temperature was controlled by a cryostat with temperature fluctuations smaller than $2 \mathrm{mK}$ (see Ref. [20] for details on the cryostat). The electrical signals were measured using a HP3458A multimeter in connection with a custom-built frequency generator as sketched in Fig. 1 (see Ref. [21] for details on the electrical setup, but notice that in this experiment we used the generator and multimeter in a slightly altered configuration). The electrical setup allows for measurements in the frequency $\left(\nu_{\mathrm{e}}\right)$ range from $1 \mathrm{mHz}$ up to $100 \mathrm{~Hz}$.

The used thermistor was of the type U23UD from Bowthorpe Thermometrics and was positioned in the middle of a sphere as shown in Fig. 2. The details of the sphere are as discussed earlier unimportant, and in this case the piezoelectric bulk transducer [15] is used as a container. The sphere is filled at room temperature with additional liquid in the reservoir. This additional liquid ensures that the sphere stays completely filled as it is cooled to the relevant temperature.

The liquid investigated was a five-ring polyphenyl ether (5-polyphenyl-4-ether), the Santovac ${ }^{\circledR} 5$ vacuum pump fluid (CAS number 2455-71-2). The liquid was used as received, without further purification.

Data were taken as frequency scans at constant temperature, with the liquid in thermal equilibrium. The direct output from the frequency generator was measured under electrical load, at each sample temperature. This eliminates any longterm drifts in the frequency generator output voltage. The

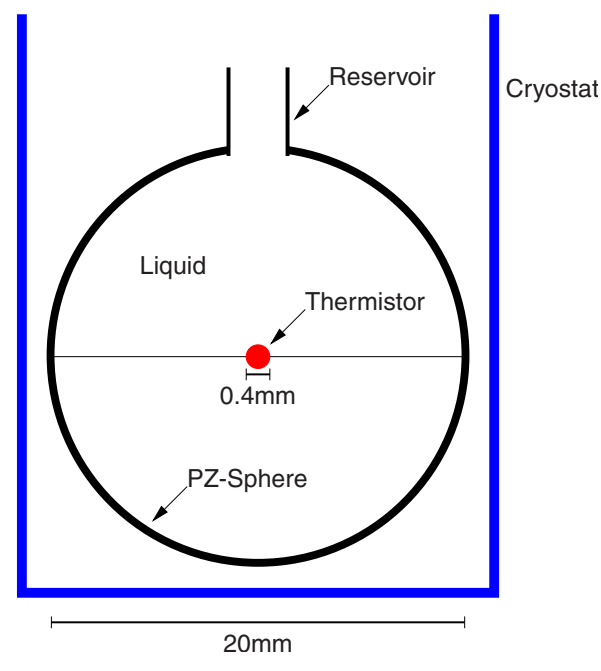

FIG. 2. (Color online) Schematic illustration of the measuring setup. The thermistor acting as combined heater and thermometer is placed in a sphere filled with the liquid. The sphere is itself placed in a cryostat.

measurements were furthermore carried out at two different input amplitudes, $\left\langle\left|U_{1}\right|\right\rangle \approx 4.9 \mathrm{~V}$ and $\left\langle\left|U_{1}\right|\right\rangle \approx 2.9 \mathrm{~V}$, allowing for a test of the inversion algorithm and linearity of the final thermal response. If not stated otherwise all results presented in this paper are from the larger of the two amplitudes. To test for reproducibility and equilibrium some of the measurements were retaken during the reheating of the sample. The outcome of the measurements are the complex amplitudes $U_{i}\left(\nu_{\mathrm{e}}\right)$ and $V_{i}\left(\nu_{\mathrm{e}}\right)$ for the harmonics of the voltages $U$ and $V$. Table I gives the key experimental parameters for the measurements at the high input amplitude.

\section{DATA ANALYSIS}

In the following we denote the measured thermal impedance $Z_{2}$ by simply $Z$, dropping the subscript 2 . The subscripts were practical in the technical description of the detection method indicating that the thermal impedance is found at a thermal frequency $\omega$, which is the double of the electrical frequency $\omega_{\mathrm{e}}$. But in discussing the thermal properties of liquids only the thermal frequency $\omega=2 \pi \nu$ is relevant. The temperature we refer to at which a measurement is done is the mean temperature at the bead surface, $T=T_{0}+T_{\text {cryo }}$.

The thermal impedance has been measured at two different input voltage amplitudes $U_{1}$ of the voltage divider in Fig. 1 and thus at two different power amplitudes. The liquid thermal response, the temperature, is expected to be linear in the power amplitudes and thus the thermal impedance should be independent of the power amplitude. The thermal impedance has been calculated from the measured voltage harmonics using both the first-order method described in Sec. IV D and using the higher-order method described in Sec. IV F.

The top part of Fig. 3 shows the frequency-dependent relative difference between the calculated thermal impedance from the two amplitudes. The results from the two methods (the first-order and higher-order methods) for three selected temperatures are shown in the figure. In the lower part the 

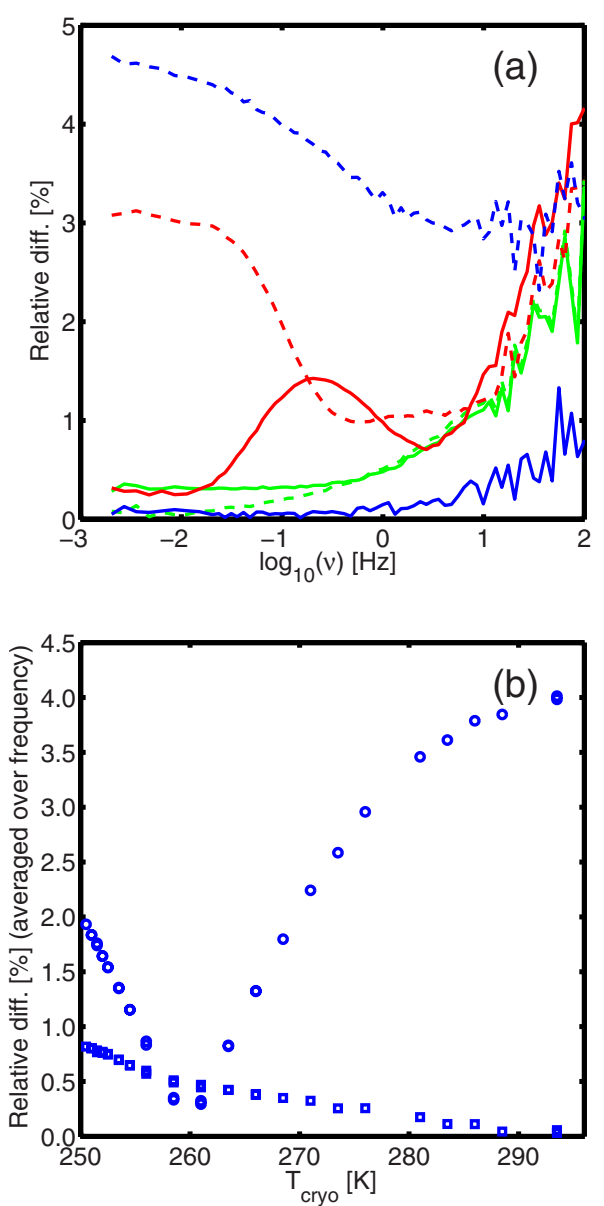

FIG. 3. (Color online) Comparison of the thermal impedance $Z$ obtained from the measurements at two different amplitudes $\left(\left\langle U_{1}\right\rangle\right.$ $=4.9$ and $2.9 \mathrm{~V}$ ), and from using the first-order model as given in Eq. (28) and higher-order model as given in Eq. (36) (as described in Secs. IV D and IV F). (a) The frequency-dependent relative difference between the two methods $\left(\left|Z_{\mathrm{amp}=2.9 \mathrm{v}}-Z_{\mathrm{amp}=4.9 \mathrm{v}}\right| /\right.$ $\left.\left|Z_{\mathrm{amp}=2.9 \mathrm{v}}\right|\right)$. Full lines: the difference for the higher-order model. Dashed lines: the difference for the first-order model. Cryostat temperatures are blue (dark gray) 293.5 K, green (light gray) $261.0 \mathrm{~K}$, and red (gray) $250.5 \mathrm{~K}$. (b) shows the frequency averaged relative difference $\left(\left\langle\left|Z_{\mathrm{amp}=2.9 \mathrm{v}}-Z_{\mathrm{amp}=4.9 \mathrm{v}}\right|\right\rangle_{\nu} /\left\langle\left|Z_{\mathrm{amp}=2.9 \mathrm{v}}\right|\right\rangle_{\nu}\right)$. Squares are the difference for the higher-order model. Circles are the difference for the first-order model.

average relative difference is shown as a function of temperature. It is seen that the higher-order method generally reduces the difference significantly. Only in a very small temperature interval does the two methods give equally good results. This demonstrates the necessity of taking into account the higher-order terms of the elaborated analysis (as described in Sec. IV F).

Figure 4 shows the real and imaginary parts of the thermal impedance $Z$ in a $\log$ - $\log$ plot at $295.6 \mathrm{~K}$. The measured impedance has more features than predicted by the simple expression for the thermal impedance of spherical effusion (9). For example the high-frequency behavior should be characterized by a line of slope $1 / 2$ but this is seen not to be the case. The additional features come from thermal properties of the thermistor bead, the influence of which we are

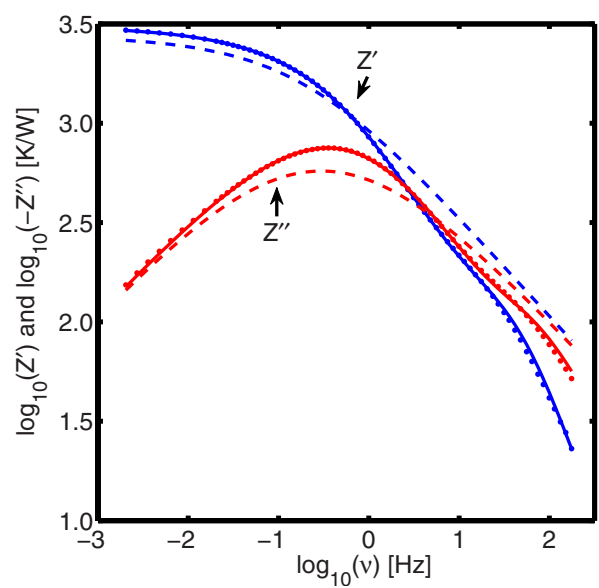

FIG. 4. (Color online) Comparison of measured thermal impedance $Z$ with the result from fitting the full model of liquid and thermistor [Eqs. (9), (42), and (43)], and the simple model of $Z_{\text {liq }}$ [Eq. (9)]. The parameters used in evaluation of the simple model are the ones found from the full analysis. Data are taken at room temperature $(T=295.6 \mathrm{~K})$. Dots: measured points. Full lines: full model of liquid and thermistor bead. Dashed lines: simple model including only the liquid.

going to study below. We stress that although $Z$ shows dispersion and has real and imaginary parts this has nothing to do with liquid relaxation-which is absent at this temperature - but is only a consequence of heat diffusion in spherical geometry. However, relaxation should of course affect the thermal impedance. This is shown in Fig. 5 where $Z$ 's at 252.8 and $256.9 \mathrm{~K}$ are compared. The glass transition is seen to give only a slight perturbation of the shape of the $Z$ curve. In order to obtain any reliable specific heat data from the thermal impedance it is thus imperative to have an accurate model of the thermal interaction between liquid and thermistor bead.

The bead (see Fig. 6) consists of a core of radius $r_{0}$, where the temperature amplitude $\delta T$ is actually measured and the heat current $P$ is generated. This core is surrounded by a

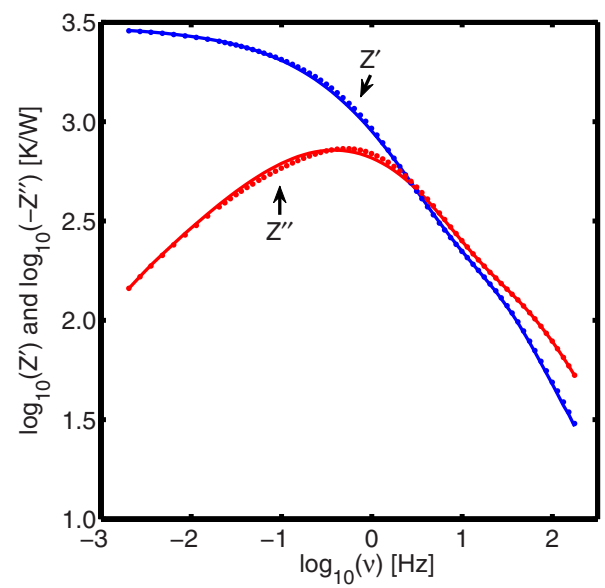

FIG. 5. (Color online) Comparison of thermal impedance $Z$ at two temperatures, showing the small difference due to the frequency-dependent specific heat of the liquid, $c_{l}(\nu)$. Lines: $T$ $=256.9$ K. Dots: $T=252.8 \mathrm{~K}$. 


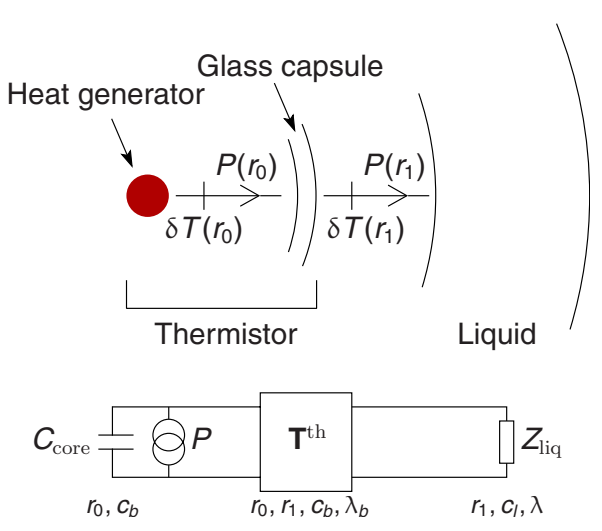

FIG. 6. (Color online) Schematic thermal network model of the thermistor bead and liquid. The thermal current $P$ and temperature $\delta T$ are indicated at the inner radius $r_{0}$ at the border between the heater and the glass capsule and at the outer radius $r_{1}$ at the border between the glass capsule and the liquid. The thermal impedance of the liquid is defined as $Z_{\mathrm{liq}}=\delta T\left(r_{1}\right) / P\left(r_{1}\right)$. The thermal impedance seen from the thermistor is $Z=\delta T\left(r_{0}\right) / P$. The two are connected by Eqs. (42) and (43), utilizing the thermal transfer matrix (C3).

glass capsule of outer radius $r_{1}$, at which the bead is in contact with the liquid. The heat flow $P\left(r_{1}\right)$ out through the surface at $r_{1}$ is in general different from the heat flow $P\left(r_{0}\right)$ out through the surface at $r_{0}$. Also the temperature $\delta T\left(r_{1}\right)$ can be different from $\delta T\left(r_{0}\right)$. Now the liquid thermal impedance is

$$
Z_{\mathrm{liq}}=\frac{\delta T\left(r_{1}\right)}{P\left(r_{1}\right)}
$$

whereas the measured thermal impedance is rather

$$
Z=\frac{\delta T}{P}
$$

The capsule layer has a heat conductivity $\lambda_{b}$ and a specific heat $c_{b}$. The bead is a solid for which in general the difference between the isobaric and isochoric specific heats is small, and they are frequency independent. The heat diffusion through this shell is thus well described [14] by a thermal transfer matrix $\mathbf{T}^{\mathrm{th}}=\mathbf{T}^{\mathrm{th}}\left(\lambda_{b}, c_{b}, r_{1}, r_{0}\right)$ (see also Appendix C),

$$
\left(\begin{array}{c}
\delta T\left(r_{1}\right) \\
P\left(r_{1}\right) /(i \omega)
\end{array}\right)=\mathbf{T}^{\mathrm{th}}\left(\begin{array}{c}
\delta T\left(r_{0}\right) \\
P\left(r_{0}\right) /(i \omega)
\end{array}\right)
$$

This implies that the thermal impedance $Z_{\mathrm{liq}}=Z_{r_{1}}$ at radius $r_{1}$ is transformed to a thermal impedance $Z_{r_{0}}$ at radius $r_{0}$ via

$$
Z_{r_{0}}=\frac{1}{i \omega} \frac{T_{12}^{\mathrm{th}}-T_{22}^{\mathrm{th}} i \omega Z_{\mathrm{liq}}}{T_{11}^{\mathrm{th}}-T_{21}^{\mathrm{th}} i \omega Z_{\mathrm{liq}}}
$$

A small part of generated power is stored in the core of the bead. The heat capacity $C_{\text {core }}$ of the core is taken to be $4 / 3 \pi r_{0}^{3} c_{b}$. The measured thermal impedance $Z$ is thus related to $Z_{r_{0}}$ by

$$
\frac{1}{Z}=i \omega C_{\mathrm{core}}+\frac{1}{Z_{r_{0}}}
$$

The model relating $Z_{\text {liq }}$ to the measured $Z$ described by Eqs. (9), (42), and (43) is depicted in the electric equivalent diagram in Fig. 6.

In order to get $Z_{\text {liq }}$ from the measured $Z$ one has to know the parameters $r_{0}, r_{1}, \lambda_{b}$, and $c_{b}$ characterizing the thermal structure of the intervening thermistor bead. This can in principle be done by fitting $Z$ of the model obtained by combining Eqs. (9), (42), and (43) to the measured frequencydependent thermal impedance. An electric equivalent network of the model is depicted in Fig. 6. This model has six frequency-independent intrinsic parameters, namely, $r_{0}$, $r_{1}, \lambda_{b}, c_{b}, \lambda$, and $c_{l}$, if the fit is limited to the temperature region where $c_{l}$ can be considered frequency independent. By careful inspection of the equations it can be found that the number of independent parameters that can be deduced from a fit is only five. The five parameters can be chosen in a number of ways, of which we have chosen

$$
\tau_{l}=r_{1}^{2} \frac{c_{l}}{\lambda}, \quad \tau_{b}=r_{1}^{2} \frac{c_{b}}{\lambda_{b}}, \quad \hat{r}=\frac{r_{0}}{r_{1}}, \quad \hat{c}=\frac{c_{l}}{c_{b}}, \quad Z_{\mathrm{liq}, 0}=\frac{1}{4 \pi \lambda r_{1}}
$$

This set of variables has the advantage that it minimizes the number of parameters which mix bead and liquid properties. Here, $\hat{c}$ is the only one such mixed parameter. The expressions for the transfer matrix and $Z_{\mathrm{liq}}$ in terms of these parameters are given in Appendix C.

The five-parameter model was fitted to the measured thermal impedance $Z$ by using a least-square algorithm. The results are shown in Figs. 7 and 8. Measurements were taken both going downward to the glass transition and upward again to room temperature. In the glass transition range where the liquid specific heat $c_{l}$ is expected to be complex and frequency dependent, these fits of course do not work. This is the reason for the scatter of data at low temperature. This difference between the high- and low-temperature regimes can also be seen directly in the error from the fit as illustrated on the lower part of Fig. 8.

The solid line in Fig. 4 shows the result of such a fit to $Z$ data at room temperature $(295.6 \mathrm{~K})$. The model captures the features in the frequency dependence of the measured thermal impedance very precisely. Also in Fig. 4 is shown the thermal impedance as it would have been if the properties of the thermistor bead itself had been negligible, i.e., if $Z$ was identical to $Z_{\text {liq }}$ of Eq. (9) We note that especially the highfrequency behaviors are different and also the limiting value at low frequency is higher for the measured $Z$ than for $Z_{\text {liq. }}$. This is due to the additional thermal resistance in the bead capsule layer.

In Table II we report the results for $\tau_{b}$ and $\hat{c}$ obtained at room temperature. Four values are given for each quantity; they are taken at the two input amplitudes and from an initial measurement at room temperature and a measurement taken at the end of the temperature scan. The method is seen to give very reproducible results. 


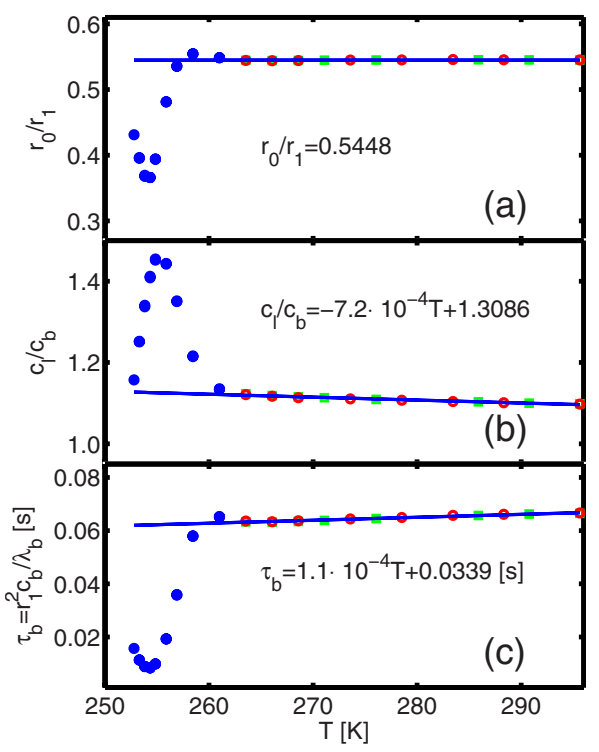

FIG. 7. (Color online) Results from fitting the measured thermal impedance $Z$ to the five-parameter thermal network model (Fig. 6) of the spherical heat effusion experiment [Eqs. (9), (42), and (43)]. This figure shows (a) $\hat{r}=r_{0} / r_{1}$, (b) $\hat{c}=c_{l} / c_{b}$, and (c) $\tau_{b}$ (the remaining parameters are shown in Fig. 8). Open squares (red): data taken going down in temperature. Open circles (green): data taken going up in temperature. Closed circles (blue): low-temperature data including points taken going down and up. Line: interpolated temperature dependencies of $\hat{r}, \hat{c}$, and $\tau_{b}$ found based on data from above the liquid glass transition temperature range, used for extrapolating down in the region where the liquid relaxation sets in (the functional form and fitting results are shown).

The five parameters are extrapolated down to the regime of frequency-dependent $c_{l}$ by the functions indicated on the figures. $\hat{r}$ is considered being temperature independent. $\hat{c}, \tau_{l}$, and $\tau_{b}$ are to a good approximation linear in temperature over the investigated temperature range. $Z_{\mathrm{liq}, 0}$ is found to be very well fitted by a second degree polynomial.

The extrapolated values of the five parameters can then be used for calculating $Z_{\text {liq }}$ from the measured thermal impedance $Z$, inverting relations (43) and (42). In Fig. 9 the calculated thermal impedance of the liquid, $Z_{\mathrm{liq}}$, and the raw measured thermal impedance $Z$ are shown for data taken at room temperature $(=295.6 \mathrm{~K})$.

As discussed earlier effusion from a plane plate can only give the effusivity $e=\sqrt{\lambda c}$. Thus, the specific heat cannot be found absolutely. In contrast effusion from a sphere is able to give both the heat conductivity and the longitudinal specific heat absolutely even if the latter is frequency dependent. However, the heat conductivity has to be frequency independent to allow for this separation. The separation is possible because $Z_{\text {liq }}$ as given in Eq. (9) has the low-frequency limiting value $Z_{\mathrm{liq}, 0}=1 /\left(4 \pi \lambda r_{1}\right)$. The convergence is rather slow involving the square root of the frequency. However, if we look at the reciprocal quantity, the thermal admittance $Y_{\text {liq }}$ $=1 / Z_{\text {liq }}$,

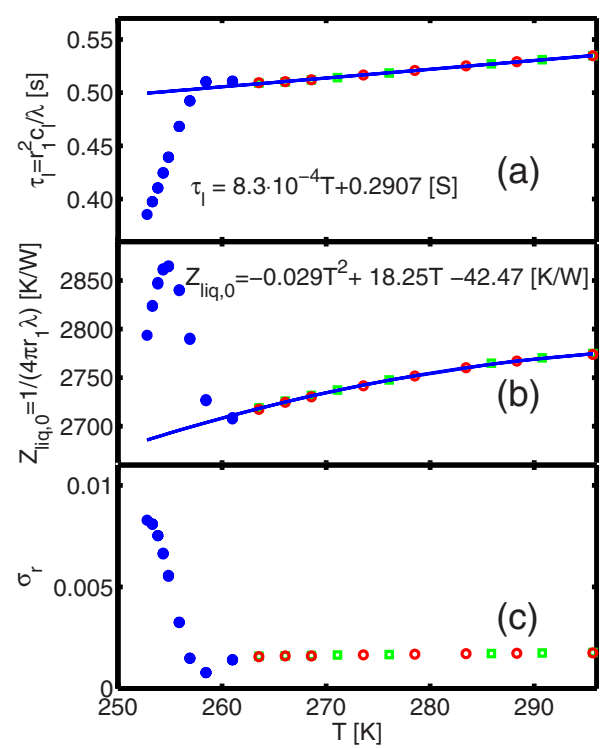

FIG. 8. (Color online) Results from fitting the measured thermal impedance $Z$ to the five-parameter thermal network model (Fig. 6) of the spherical heat effusion experiment [Eqs. (9), (42), and (43)]. This figure shows (a) $\tau_{l}$, (b) $Z_{\mathrm{liq}, 0}$, and (c) the relative error from the fit, $\sigma_{r}=\sqrt{\Sigma_{\nu}\left|Z-Z_{\text {model }}\right|^{2} / \Sigma_{\nu}\left|Z_{\text {model }}\right|^{2}}$ (the remaining parameters are shown in Fig. 7). Open squares (red): data taken going down in temperature. Open circles (green): data taken going up in temperature. Closed circles (blue): low-temperature data including points taken going down and up. Line: interpolated temperature dependencies of $\tau_{l}$, and $Z_{\text {liq }, 0}$ found based on data from above the liquid glass transition temperature range, used for extrapolating down in the region where the liquid relaxation sets in (the functional form and fitting results are shown).

$$
Y_{\text {liq }}=4 \pi \lambda r_{1}\left(1+\sqrt{i \omega r_{1}^{2} c_{l} / \lambda}\right),
$$

we observe that when $c_{l}$ is frequency independent the squareroot frequency term can be canceled by taking the difference between $Y_{\text {liq }}^{\prime}$ and $Y_{\text {liq, }}^{\prime \prime}$

$$
\lambda=\frac{Y_{\text {liq }}^{\prime}-Y_{\text {liq }}^{\prime \prime}}{4 \pi r_{1}} .
$$

This is valid even in the glass transition range at sufficiently low frequencies where $c_{l}$ reaches its noncomplex equilibrium value. Finally, using the found values for $\lambda$ the frequencydependent longitudinal specific heat is calculated from

$$
c_{l}(\omega)=\frac{\lambda}{i \omega r_{1}^{2}}\left(\frac{Y_{\text {liq }}}{4 \pi \lambda r_{1}}-1\right)^{2} .
$$

However, the structure of the five-parameter model only allows for determining $\hat{r}$ but not $r_{0}$ and $r_{1}$ independently from the fit to data. To obtain the absolute value of $\lambda$ and $c_{l}$ one of the radii is needed. Measured values of $r_{1}$ are given in Table I. As indicated the bead is not completely spherical, and it is therefore needed to determine which value of $r_{1}$ to use.

According to the specifications for Santovac ${ }^{\circledR} 5$ given by Scientific Instrument Service, Inc. [22] the value of $\lambda$ at $20{ }^{\circ} \mathrm{C}$ is $0.1330 \mathrm{~W} / \mathrm{K} \mathrm{m}$. They likewise report the isobaric specific heat per mass $c_{p} / \rho=0.35 \mathrm{cal} / \mathrm{g} \mathrm{K}$ and density $\rho$ 
TABLE II. Heat conductivity and specific heat for the liquid $\left(\lambda\right.$ and $\left.c_{l}\right)$, as defined in relation to Figs. 10 and 12. Fitting parameters characterizing the bead $\left(\tau_{b}\right.$ and $\left.\hat{c}\right)$ from the fits to the five-parameter mode. The results are at room temperature $(295.6 \mathrm{~K})$ and taken using two input amplitudes $\left|U_{1}\right|$. The two scans are the initial scan and a scan taken after the temperature scan coming back to room temperature. The results indicate the reproducibility of the method.

\begin{tabular}{ccccccc}
\hline \hline $\begin{array}{c}\left|U_{1}\right| \\
(\mathrm{V})\end{array}$ & $\begin{array}{c}P_{2} \\
(\mathrm{~mW})\end{array}$ & Scan & $\begin{array}{c}\tau_{b} \\
(\mathrm{~s})\end{array}$ & $\hat{c}=c_{l} / c_{b}$ & $\begin{array}{c}\lambda \\
(\mathrm{W} / \mathrm{K} \mathrm{m})\end{array}$ & $\begin{array}{c}c_{l} \\
\left(10^{6} \mathrm{~J} / \mathrm{K} \mathrm{m}^{3}\right)\end{array}$ \\
\hline \multirow{2}{*}{4.9} & 0.63 & 1 & 0.0665 & 1.0983 & 0.13759 & 1.7597 \\
& & 2 & 0.0666 & 1.0977 & 0.13761 & 1.7602 \\
2.9 & 0.23 & 1 & 0.0663 & 1.0989 & 0.13786 & 1.7627 \\
& & 2 & 0.0668 & 1.0953 & 0.13782 & 1.7623 \\
\hline \hline
\end{tabular}

$=1.204 \mathrm{~g} / \mathrm{cm}^{3}$ at $20{ }^{\circ} \mathrm{C}$, giving a specific heat per volume of $c_{p}=1.76 \times 10^{6} \mathrm{~J} / \mathrm{K} \mathrm{m}^{3}$. At these temperatures the isobaric and longitudinal specific heats are identical; we have therefore chosen to use a value for $r_{1}$ which matches $c_{l}$, within the errors, to the reference value. This "optimized" value is very

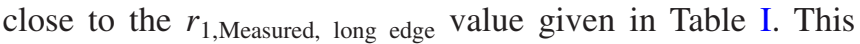
choice gives a value of $\lambda$ which is $3.5 \%$ too big compared to the literature value.

Figure 10 shows how the temperature dependence of the heat conductivity $\lambda$ of the sample liquid is found. In Fig. 11 is shown the final frequency-dependent specific heat for a number of temperatures. Table II reports the roomtemperature values for $c_{l}$ and $\lambda$ for two temperature amplitudes, and data reproduced after a temperature scan down and up again. The values indicate the reproducibility of the method.

\section{DISCUSSION}

The derived longitudinal specific heat displayed in Fig. 11 shows the expected relaxation phenomena at the glass tran-

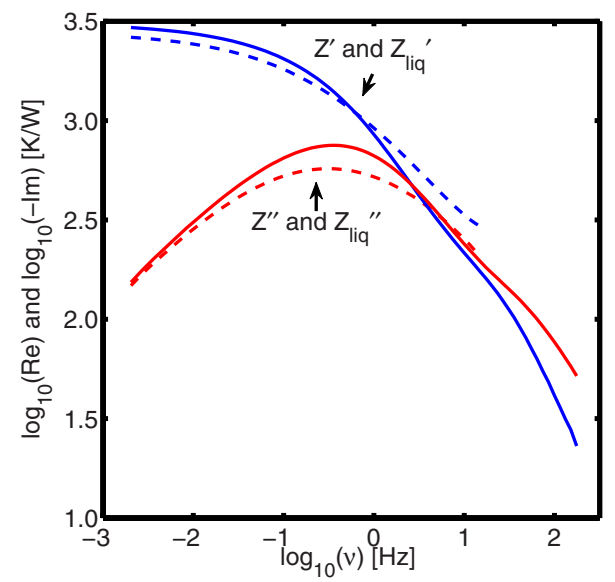

FIG. 9. (Color online) Comparison of the raw measured thermal impedance $Z$ and final liquid thermal impedance $Z_{\text {liq }}$ corrected for the influence of the thermistor encapsulation. Data are taken at room temperature $(T=295.6 \mathrm{~K})$ and are equivalent to the data shown in Fig. 4. The liquid thermal impedance is truncated at high frequencies $\left(\nu>10^{1.2} \mathrm{~Hz}\right)$ as the bead becomes dominant at higher frequencies, leading to unphysical behavior of the calculated liquid impedance. Full lines: raw thermal impedance $Z$. Dashed lines: liquid thermal impedance $Z_{\text {liq }}$. sition. At $252.8 \mathrm{~K} c_{l}$ decreases from $c_{0}=c_{l}(\omega \rightarrow 0)=1.75$ $\times 10^{6} \mathrm{~J} / \mathrm{K} \mathrm{m}^{3}$ to $c_{\infty}=c_{l}(\omega \rightarrow \infty)=1.30 \times 10^{6} \mathrm{~J} / \mathrm{K} \mathrm{m}^{3}$. The temperature dependency of $c_{0}$ above the glass transition range is shown in Fig. 12. The temperature dependency of the characteristic relaxation time $\tau$ is shown in Fig. 13. The high-temperature data are seen to show spurious frequency dependency in both the real and imaginary parts of $c_{l}$. This may be caused by small systematic errors in the data processing or data acquisition that we have not been able to trace yet. It may also be due to the small eccentricity of the
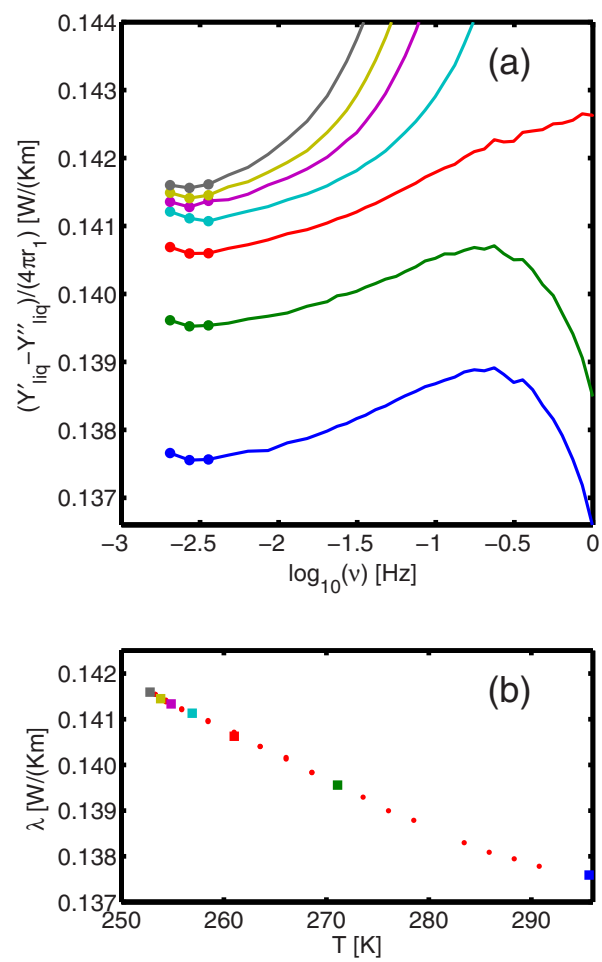

FIG. 10. (Color online) Thermal conductivity of the liquid. (a) $\left(Y_{\text {liq }}^{\prime}-Y_{\text {liq }}^{\prime \prime}\right) / 4 \pi r_{1}$ as a function of frequency, plotted for a selection of temperatures (same temperatures as in Fig. 11, with colors indicating same data sets). According to Eq. (46) the thermal conductivity is the low-frequency limit of this quantity. The points on the lines indicate the data points used for defining the low-frequency limit (taken as an average over these points). (b) Thermal conductivity of the liquid defined from the average over the low-frequency limit of $\left(Y_{\text {liq }}^{\prime}-Y_{\text {liq }}^{\prime \prime}\right) / 4 \pi r_{1}$. Squares correspond to the curves shown on the upper figure and dots correspond to the remaining data sets. 


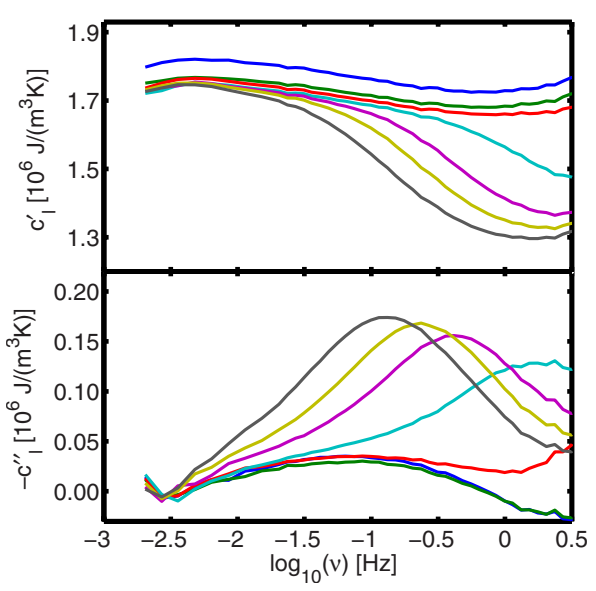

FIG. 11. (Color online) Real and imaginary parts of the longitudinal specific heat of the liquid $c_{l}$ at a number of the investigated temperatures. The frequency axis is truncated at $0.5 \mathrm{~Hz}$ as the signal above this frequency is dominated by the inner structure of the thermistor. Temperatures are 295.6, 271.1, 261.0, 256.9, 254.9, 253.8 , and $252.8 \mathrm{~K}$.

bead. Due to the functional relationship [Eq. (9)] between $Z_{\text {liq }}$ and $c_{l}$ a small relative error $\left|d Z_{\text {liq }} / Z_{\text {liq }}\right|$ in the thermal impedance may propagate to a large relative error $\left|d c_{l} / c_{l}\right|$ in the specific heat. As a function of the dimensionless Laplace frequency $s=i \omega \tau_{l}$, one finds

$$
\frac{d c_{l}}{c_{l}} / \frac{d Z_{\mathrm{liq}}}{Z_{\mathrm{liq}}}=2 \frac{1+\sqrt{s}}{\sqrt{s}} .
$$

This factor is 4 at the characteristic heat diffusion frequency $(s=1)$ increasing to a factor of 22 at 0.01 times this frequency. Thus, the specific heat is difficult to get reliable more than 2 decades below the characteristic diffusion frequency. On the other hand 1 decade above this frequency the measured $Z$ is dominated by the thermal structure of the

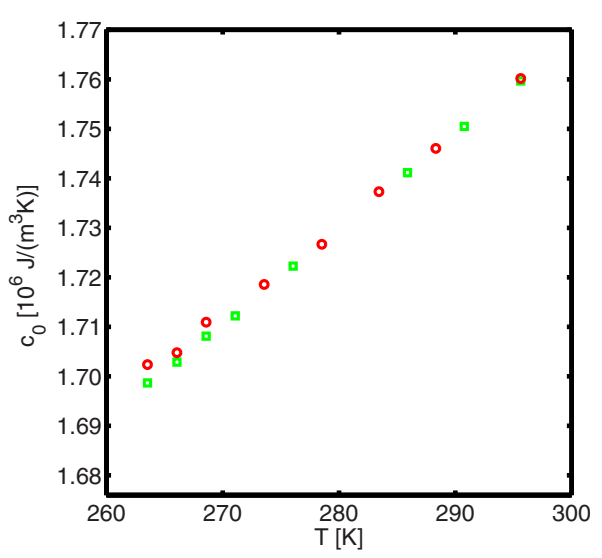

FIG. 12. (Color online) dc specific heat $c_{0}$ of the liquid for temperatures above the liquid glass transition temperature range. The specific heat is found as the average over the real part of $c_{l}$ in the frequency range $10^{-2}-1 \mathrm{~Hz} . c_{0}$ corresponds to the dc value of $c_{p}$ as can be seen from Eqs. (3) and (4). Open squares (green): data taken going down in temperature. Open circles (red): data taken going up in temperature.

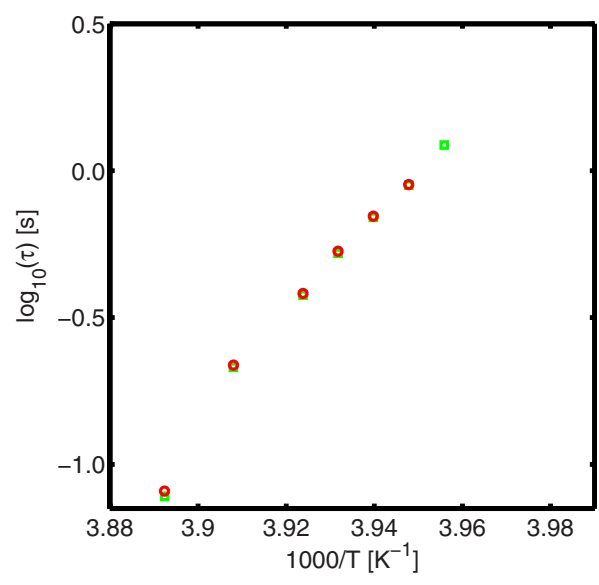

FIG. 13. (Color online) Characteristic relaxation time $\tau$ for the longitudinal specific heat $c_{l}(\omega)$. The time is found from a fit to 2-order polynomial to a few points around the maximum of the imaginary part of $c_{l}$ and corresponds to $\tau=1 /\left(2 \pi \nu_{\mathrm{lp}}\right)$, where $\nu_{\mathrm{lp}}$ is the frequency of maximum loss. Open squares (green): data taken going down in temperature. Open circles (red): data taken going up in temperature.

thermistor bead that can only to some extent be reliably modeled.

\section{CONCLUSION}

From a theoretical point of view determination of the frequency-dependent specific heat by a thermal effusion method based on a sphere has many ideal features. The surface of a sphere-being closed-has no boundary and thus no associated boundary effects like a finite plane plate. The thermomechanical coupling problem can be treated analytically, i.e., the influence of the increasing dynamic shear modulus and the mechanical boundary conditions are adequately taken into account. Although the thermomechanical problem can also be solved in the one-dimensional unilateral case, it is not so obvious whether it really applies to the experimental finite plate realizations. It is of course preferable if thermal experiments can give two independent thermal properties, e.g., the specific heat and the heat conductivity or the effusivity and diffusivity. Effusion experiments in different geometries give the effusivity only, unless a characteristic length scale comes into play with the heat diffusion length. For the infinite plane there is no such length scale whereas in the spherical case this length scale is the radius of the heat producing sphere. This advantage is however also the weakness of the method since it limits the practical frequency window to be studied.

\section{ACKNOWLEDGMENTS}

The authors thank Professor Jeppe C. Dyre for supporting this work. This work was supported by the Danish National Research Foundation (DNRF) Center for Viscous Liquid Dynamics "Glass and Time."

\section{APPENDIX A: THE ac METHOD TO HIGHER ORDER}

In this appendix we analyze which higher-order terms must be included in order to get a relative accuracy of 1 


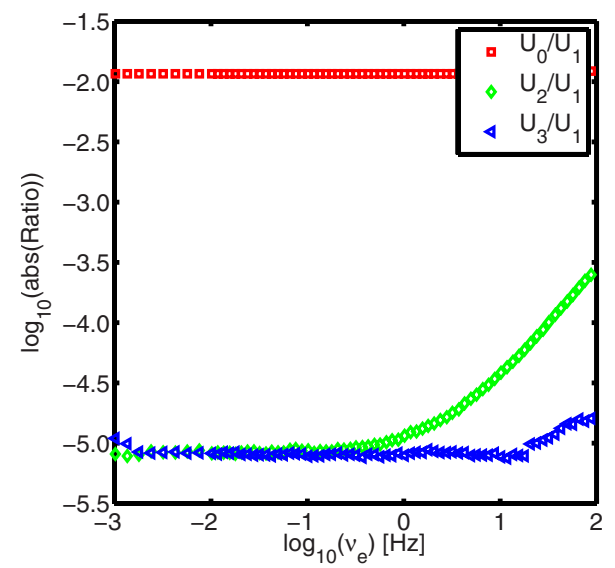

FIG. 14. (Color online) Relative magnitude of the components of input voltage $U$ to the dominant $U_{1}$ component; $\left\langle\left|U_{1}\right|\right\rangle$ $=4.909$ V. See also Table III.

$\times 10^{-4}$ on the measured temperature amplitude from the Fourier components of the measured voltages of the voltage divider (see Fig. 1). In the following we will include up to fourth harmonics in all the relevant quantities; hence, we write

$$
\begin{aligned}
& V(t)=\frac{1}{2}\left(V_{0}+V_{1} E_{1}+V_{2} E_{2}+V_{3} E_{3}+V_{4} E_{4}+\text { c.c. }\right), \\
& U(t)=\frac{1}{2}\left(U_{0}+U_{1} E_{1}+U_{2} E_{2}+U_{3} E_{3}+U_{4} E_{4}+\text { c.c. }\right), \\
& P(t)=\frac{1}{2}\left(P_{0}+P_{1} E_{1}+P_{2} E_{2}+P_{3} E_{3}+P_{4} E_{4}+\text { c.c. }\right), \\
& \Delta T(t)=\frac{1}{2}\left(T_{0}+T_{1} E_{1}+T_{2} E_{2}+T_{3} E_{3}+T_{4} E_{4}+\text { c.c. }\right) .
\end{aligned}
$$

Combining the expansion of the thermistor resistance to second order [Eq. (34)], with the voltage divider equation [Eq. (16)], the voltage across the preresistor becomes, to second order in $\Delta T$,

$$
V(t)=\frac{1}{A+1}\left(1-a \Delta T+b \Delta T^{2}\right) U
$$

with $A=R_{0} / R_{\text {pre }}, \quad a=A \alpha_{1} /(1+A), \quad$ and $\quad b=\left[A \alpha_{1} /(1+A)\right]^{2}$ $-A \alpha_{2} /(1+A)$.

If this expression is explicitly calculated using the above expansions of $U$ and $T$ [Eqs. (A1b) and (A1d)] a large number of terms are obtained. In the following we make a numerical inspection of which terms are most significant, in a worst case, to reduce the number of terms included.

In order to calculate the order of magnitude of the terms, an estimate of the size of the components of $U$ and $\Delta T$ is needed. In Fig. 14 we show the relative magnitudes of the different components of the input voltage $U$ of the voltage divider to $U_{1}$. In Table III we summarize the upper limits of these ratios. In Fig. 15 we show the relative magnitude of the different components of the power calculated from the mea-
TABLE III. Maximum of relative magnitude of the components of input voltage $U$ to the dominant first harmonic $U_{1}$, as shown in Fig. 14 (for $\nu<40 \mathrm{~Hz}$ ).

\begin{tabular}{cccc}
\hline \hline$U_{0} / U_{1}$ & $U_{2} / U_{1}$ & $U_{3} / U_{1}$ & $U_{4} / U_{1}{ }^{\mathrm{a}}$ \\
\hline $2 \times 10^{-2}$ & $10^{-4}$ & $10^{-5}$ & $3 \times 10^{-5}$
\end{tabular}

$\overline{\overline{\mathrm{a}} U_{4} / U_{1} \text { was estimated from an additional measurement where } U_{4}}$ was included.

sured $U_{i}$ and $V_{i}$ components. In Table IV we summarize the upper limits on these ratios. We estimate the relative size of the temperature amplitudes as equal to the relative sizes of the power amplitudes. In Table $\mathrm{V}$ we give characteristic values of the quantities describing the thermistor.

Combing the estimates given in Table V, with Eq. (26), we observe that a change in $T_{2}$ of $1 \times 10^{-4} \mathrm{~K}$ corresponds to a change in $V_{3} / U_{1}$ of $5 \times 10^{-7}$. Hence, only terms in the expansion of Eq. (A2) which are larger than $5 \times 10^{-7} U_{1}$ will be included.

By explicit substitution of Eqs. (A1b) and (A1d) into Eq. (A2) and insertion of the estimates from Tables III-V the following expressions are found for the first and third harmonics on the voltage $V$ when disregarding terms smaller than $5 \times 10^{-7} U_{1}$ :

$$
\begin{gathered}
V_{1}=\frac{1}{A+1}\left[U_{1}-a T_{0} U_{1}-\frac{1}{2} a T_{2} U_{1}^{*}+X_{1}\right], \\
V_{3}=\frac{1}{A+1}\left[-\frac{1}{2} a T_{2} U_{1}+U_{3}+X_{3}\right],
\end{gathered}
$$

with

$$
X_{1}=-a T_{1} U_{0}+b T_{0}^{2} U_{1}+\frac{1}{2} b T_{2} T_{2}^{*} U_{1}+b T_{0} T_{2} U_{1}^{*},
$$

$$
X_{3}=-\frac{1}{2} a T_{4} U_{1}^{*}+\frac{1}{4} b T_{2}^{2} U_{1}^{*}+b T_{0} T_{2} U_{1}+b T_{0} T_{4} U_{1}^{*} .
$$

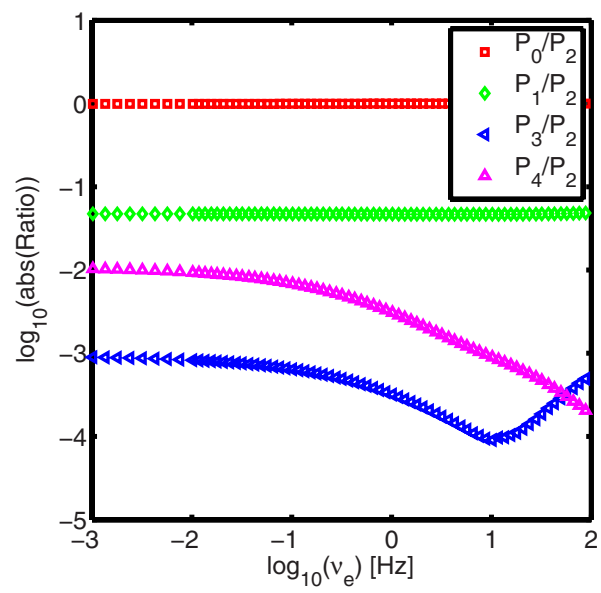

FIG. 15. (Color online) Magnitude of the components of the power to the dominant $P_{2}$ component; $T_{\text {cryo }}=256.9 \mathrm{~K}$. See also Table IV. 
TABLE IV. Maximum of relative magnitude of the components of the power as shown in Fig. 15 (for $\nu<40 \mathrm{~Hz}$ ).

\begin{tabular}{cccc}
\hline \hline$P_{0} / P_{2}$ & $P_{1} / P_{2}$ & $P_{3} / P_{2}$ & $P_{4} / P_{2}$ \\
\hline 1 & $10^{-1}$ & $10^{-3}$ & $10^{-2}$ \\
\hline
\end{tabular}

\section{APPENDIX B: POWER TERMS TO HIGHER ORDER}

The relevant set of power components is calculated from Eq. (30) as

$$
P=\frac{(U-V) V}{R_{\mathrm{pre}}}
$$

using the expansions in Eqs. (A1a) and (A1b) to be

$$
\begin{aligned}
P_{0}= & \frac{1}{R_{\mathrm{pre}}}\left(W_{0} V_{0}+\frac{1}{2} \operatorname{Re}\left(W_{1} V_{1}^{*}+W_{2} V_{2}^{*}+W_{3} V_{3}^{*}+W_{4} V_{4}^{*}\right)\right), \\
P_{1}= & \frac{1}{R_{\mathrm{pre}}}\left(W_{1} V_{0}+V_{1} W_{0}+\frac{1}{2}\left(W_{2} V_{1}^{*}+W_{1}^{*} V_{2}+W_{3} V_{2}^{*}+W_{2}^{*} V_{3}\right.\right. \\
& \left.\left.+W_{3}^{*} V_{4}+W_{4} V_{3}^{*}\right)\right), \\
P_{2}= & \frac{1}{R_{\mathrm{pre}}}\left(W_{0} V_{2}+W_{2} V_{0}+\frac{1}{2}\left(W_{1} V_{1}+W_{1}^{*} V_{3}+W_{2}^{*} V_{4}+W_{3} V_{1}^{*}\right.\right. \\
& \left.\left.+W_{4} V_{2}^{*}\right)\right), \\
P_{3}= & \frac{1}{R_{\mathrm{pre}}}\left(W_{0} V_{3}+W_{3} V_{0}+\frac{1}{2}\left(W_{1} V_{2}+W_{2} V_{1}+W_{1}^{*} V_{4}\right.\right. \\
& \left.\left.+W_{4} V_{1}^{*}\right)\right), \\
P_{4} & \frac{1}{R_{\mathrm{pre}}}\left(W_{0} V_{4}+W_{4} V_{0}+\frac{1}{2}\left(W_{1} V_{3}+W_{2} V_{2}+W_{3} V_{1}\right)\right),
\end{aligned}
$$

(B1e)

where $W=U-V$.
TABLE V. Characteristic sizes of the parameters describing the used thermistor at $T_{\text {cryo }}=256.9 \mathrm{~K}$.

\begin{tabular}{ccc}
\hline \hline$A$ & $\alpha_{1}$ & $\alpha_{2}$ \\
\hline$\approx 2$ & $\approx 4 \times 10^{-2} \mathrm{~K}^{-1}$ & $\approx 10^{-3} \mathrm{~K}^{-2}$ \\
\hline \hline
\end{tabular}

\section{APPENDIX C: THE "FIVE-PARAMETER" FORMULATION} OF THE THERMAL TRANSFER MODEL

Equations (9), (41), and (42) are in the following written in terms of the five variables,

$$
\tau_{l}=r_{1}^{2} \frac{c_{l}}{\lambda}, \quad \tau_{b}=r_{1}^{2} \frac{c_{b}}{\lambda_{b}}, \quad \hat{r}=\frac{r_{0}}{r_{1}}, \quad \hat{c}=\frac{c_{l}}{c_{b}}, \quad Z_{\mathrm{liq}, 0}=\frac{1}{4 \pi \lambda r_{1}} .
$$

The thermal impedance [Eq. (9)] is given as

$$
Z_{\text {liq }}=Z_{\text {liq }, 0} \frac{1}{\left(1+\sqrt{i \omega \tau_{l}}\right)} .
$$

The components of the thermal transfer matrix [14] in Eq. (41) are given as

$$
T_{11}^{\mathrm{th}}=\hat{r} \cosh \left[\sqrt{i \omega \tau_{b}}(1-\hat{r})\right]+\frac{1}{\sqrt{i \omega \tau_{b}}} \sinh \left[\sqrt{i \omega \tau_{b}}(1-\hat{r})\right],
$$

$$
\begin{aligned}
& T_{12}^{\mathrm{th}}=-Z_{\mathrm{liq}, 0} \frac{\hat{c}}{\tau_{l}} \frac{\sqrt{i \omega \tau_{b}}}{\hat{r}} \sinh \left[\sqrt{i \omega \tau_{b}}(1-\hat{r})\right], \\
& T_{21}^{\mathrm{th}=} \frac{1}{Z_{\mathrm{liq}, 0}} \frac{\tau_{l}}{\hat{c}} \frac{1}{\left(\sqrt{i \omega \tau_{b}}\right)^{3}}\left\{\left(1-i \omega \tau_{b} \hat{r}\right) \sinh \left[\sqrt{i \omega \tau_{b}}(1-\hat{r})\right]\right. \\
&\left.-\sqrt{i \omega \tau_{b}}(1-\hat{r}) \cosh \left[\sqrt{i \omega \tau_{b}}(1-\hat{r})\right]\right\} \\
& T_{22}^{\mathrm{th}}= \frac{1}{\hat{r}} \cosh \left[\sqrt{i \omega \tau_{b}}(1-\hat{r})\right]-\frac{1}{\hat{r} \sqrt{i \omega \tau_{b}}} \sinh \left[\sqrt{i \omega \tau_{b}}(1-\hat{r})\right] .
\end{aligned}
$$

[1] G. Harrison, The Dynamic Properties of Supercooled Liquids (Academic, New York, 1976).

[2] R. O. Davies and G. O. Jones, Adv. Phys. 2, 370 (1953).

[3] N. O. Birge and S. R. Nagel, Phys. Rev. Lett. 54, 2674 (1985).

[4] T. Christensen, J. Phys. (Paris), Colloq. 46, C8-635 (1985).

[5] T. Christensen and N. B. Olsen, J. Non-Cryst. Solids 235-237, 296 (1998).

[6] H. Huth, A. A. Minakov, A. Serghei, F. Kremer, and C. Schick, Eur. Phys. J. Spec. Top. 141, 153 (2007).

[7] N. O. Birge, Phys. Rev. B 34, 1631 (1986).
[8] I. K. Moon, Y. H. Jeong, and S. I. Kwun, Rev. Sci. Instrum. 67, 29 (1996).

[9] N. O. Birge, P. K. Dixon, and N. Menon, Thermochim. Acta 304-305, 51 (1997).

[10] A. A. Minakov, S. A. Adamovsky, and C. Schick, Thermochim. Acta 377, 173 (2001).

[11] A. A. Minakov, S. A. Adamovsky, and C. Schick, Thermochim. Acta 403, 89 (2003).

[12] T. Christensen, N. B. Olsen, and J. C. Dyre, Phys. Rev. E 75, 041502 (2007). 
[13] T. Christensen, N. B. Olsen, and J. C. Dyre, in 5th International Workshop on Complex Systems, AIP Conf. Proc. No. 982, edited by M. Tokuyama, I. Oppenheim, and H. Nishiyama (AIP, Melville, NY, 2008), p. 139.

[14] T. Christensen and J. C. Dyre, Phys. Rev. E 78, 021501 (2008).

[15] T. Christensen and N. B. Olsen, Phys. Rev. B 49, 15396 (1994).

[16] H. S. Carslaw and J. C. Jaeger, Conduction of Heat in Solids (Clarendon Press, Oxford, 1959).

[17] L. D. Landau and E. M. Lifshitz, Theory of Elasticity, 3rd ed. (Pergamon, London, 1986).

[18] N. O. Birge and S. R. Nagel, Rev. Sci. Instrum. 58, 1464 (1987).

[19] The dc value of the thermal impedance, $Z_{0}$, can be estimated from the low-frequency limit of the ac-thermal impedance at $2 \omega_{\mathrm{e}}, Z_{2}$. Our lowest frequency of measurement is $10^{-3} \mathrm{~Hz}$ at which the thermal wavelength is still shorter than the sample size (we are still in the thermally thick limit). However, the dc impedance does see the outer boundary, as it has "infinite" time to reach steady state. To compensate for this we add a small frequency, temperature, and amplitude-independent correction term to the limiting value of $Z_{2}$ when estimating $Z_{0}$. This correction term value was chosen, so that measurements with different amplitudes give the same values for $A(T)$.

[20] B. Igarashi, T. Christensen, E. H. Larsen, N. B. Olsen, I. H. Pedersen, T. Rasmussen, and J. C. Dyre, Rev. Sci. Instrum. 79, 045105 (2008).

[21] B. Igarashi, T. Christensen, E. H. Larsen, N. B. Olsen, I. H. Pedersen, T. Rasmussen, and J. C. Dyre, Rev. Sci. Instrum. 79, 045106 (2008).

[22] Scientific Instrument Service, Inc., Specifications for Santovac 5 and Santovac 5p Ultra, 2008; http://www.sisweb.com/ catalog/08/F14 